\title{
A STUDY ON $\phi$-SYMMETRIC $\tau$-CURVATURE TENSOR IN K-CONTACT MANIFOLD
}

\author{
G. Ingalahalli, C.S. Bagewadi
}

ABstract. The aim of this paper is the study of curvature properties for globally $\phi$ - $\tau$-symmetric and $\tau$-Ricci $\eta$-parallel K-contact manifolds.

2010 Mathematics Subject Classification: 53C15, 53C25, 53D15.

Keywords: Curvature tensor, K-contact manifold, Einstein, $\phi$-symmetric, space of constant curvature.

\section{INTRODUCTION}

The notion of local symmetry of a Riemannian manifold has been weakened by many authors in several ways to a different extent. In the context of contact geometry the notion of $\phi$-symmetry is introduced and studied by E. Boeckx, P. Buecken and L. Vanhecke [3] with several examples. As a weaker version of local symmetry, T. Takahashi [14] introduced the notion of locally $\phi$-symmetry on a Sasakian manifold.

In [9] M.M. Tripathi and et.al. introduced the $\tau$-curvature tensor which consists of known curvatures like conformal, concircular, projective, $M$-projective, $W_{i^{-}}$ curvature tensor $(i=0, \ldots, 9)$ and $W_{j}^{*}$-curvature tensor $(j=0,1)$. Futher, in [10], [11] M.M. Tripathi and et.al. studied $\tau$-curvature tensor in K-contact, Sasakian and Semi-Riemannian manifolds. Later in [12] the authors studied some properties of $\tau$-curvature tensor and they obtained some interesting results.

Motivated by all these works in this paper we study the globally $\phi$-Symmetric $\tau$-curvature tensor in K-contact manifold.

The $\tau$-curvature tensor is given by ([10], [11])

$$
\begin{aligned}
\tau(X, Y) Z & =a_{0} R(X, Y) Z+a_{1} S(Y, Z) X+a_{2} S(X, Z) Y+a_{3} S(X, Y) Z \\
& +a_{4} g(Y, Z) Q X+a_{5} g(X, Z) Q Y+a_{6} g(X, Y) Q Z \\
& +a_{7} r[g(Y, Z) X-g(X, Z) Y]
\end{aligned}
$$


G. Ingalahalli, C.S. Bagewadi - A study on $\phi$-symmetric $\tau$-curvature tensor ...

where $a_{0}, \ldots, a_{7}$ are some smooth functions on $M$. For different values of $a_{0}, \ldots, a_{7}$ the $\tau$-curvature tensor reduces to the curvature tensor $R$, Quasi-Conformal curvature tensor, Conformal curvature tensor, Conharmonic curvature tensor, Concircular curvature tensor, Pseudo-projective curvature tensor, Projective curvature tensor,

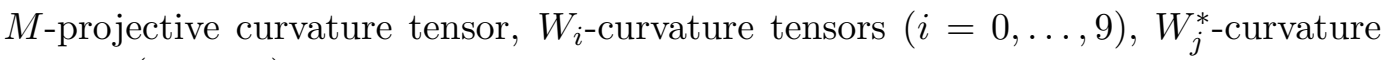
tensors $(j=0,1)$.

\section{PReliminaries}

A $(2 n+1)$-dimensional manifold $M$ is said to be an almost contact metric structure $(\phi, \xi, \eta, g)$ if it carries a tensor field $\phi$ of type $(1,1)$, a vector field $\xi, 1$-form $\eta$ and a Riemannian metric $g$ on $M$ satisfy,

$$
\begin{aligned}
& \phi^{2}=-I+\eta \otimes \xi, \quad \eta(\xi)=1, \quad \eta \circ \phi=0, \quad \phi \xi=0, \\
& g(\phi X, \phi Y)=g(X, Y)-\eta(X) \eta(Y), \quad g(X, \xi)=\eta(X) .
\end{aligned}
$$

Thus a manifold $M$ equipped with this structure is called an almost contact metric manifold $(M, \phi, \xi, \eta, g)$.

If on $(M, \phi, \xi, \eta, g)$ the exterior derivative of 1 -form $\eta$ satisfies,

$$
d \eta(X, Y)=g(X, \phi Y),
$$

then the manifold is said to a contact metric manifold.

If the contact metric structure is normal then it is called a Sasakian structure. Note that an almost contact metric manifold defines Sasakian structure if and only if,

$$
\left(\nabla_{X} \phi\right) Y=g(X, Y) \xi-\eta(Y) X,
$$

where $\nabla$ denotes the Riemannian connection on $M$. Contact metric manifold with structure tensor $(\phi, \xi, \eta, g)$ in which the Killing vector field $\xi$ satisfies the condition $\nabla_{\xi} \xi=0$, then $M$ is called the $K$-contact manifold.

In a $(2 n+1)$-dimensional K-contact manifold the following relations hold:

$$
\begin{aligned}
\nabla_{X} \xi & =-\phi X, \\
g(R(\xi, X) Y, \xi) & =g(X, Y)-\eta(X) \eta(Y), \\
R(X, Y) \xi & =\eta(Y) X-\eta(X) Y, \\
R(\xi, X) \xi & =-X+\eta(X) \xi, \\
S(X, \xi) & =2 n \eta(X), \\
S(\phi X, \phi Y) & =S(X, Y)-2 n \eta(X) \eta(Y),
\end{aligned}
$$

where $R$ and $S$ are the Riemannian curvature and the Ricci tensor of $M$, respectively. 
G. Ingalahalli, C.S. Bagewadi - A study on $\phi$-symmetric $\tau$-curvature tensor ...

Definition 1. A K-contact manifold $M$ is said to be locally $\phi$-symmetric if

$$
\phi^{2}\left(\left(\nabla_{W} R\right)(X, Y) Z\right)=0
$$

for all vector fields $X, Y, Z$ and $W$ which are orthogonal to $\xi$. The notion was introduced by T. Takahashi [14] for Sasakian manifolds.

Definition 2. A K-contact manifold $M$ is said to be globally $\phi$-symmetric if

$$
\phi^{2}\left(\left(\nabla_{W} R\right)(X, Y) Z\right)=0
$$

for all arbitrary vector fields $X, Y, Z$ and $W$ on $M$.

Definition 3. A K-contact manifold $M$ is said to be globally $\phi$ - $\tau$-symmetric if

$$
\phi^{2}\left(\left(\nabla_{W} \tau\right)(X, Y) Z\right)=0
$$

for all arbitrary vector fields $X, Y, Z, W$ and $\tau$ is the curvature tensor.

Definition 4. The Ricci tensor of a K-contact manifold is said to be $\eta$-parallel if it satisfies

$$
\left(\nabla_{X} S\right)(\phi Y, \phi Z)=0
$$

for all vector fields $X, Y, Z$. This notion of Ricci $\eta$-parallelity was first introduced by M. Kon [7] in a Sasakian manifold

\section{Globally $\phi$-Symmetric K-Contact manifold}

In this section, we define globally $\phi$-symmetric K-contact manifold. From (2) and (13), we have

$$
-\left(\left(\nabla_{W} R\right)(X, Y) Z\right)+\eta\left(\left(\nabla_{W} R\right)(X, Y) Z\right) \xi=0 .
$$

We know that

$$
g\left(\left(\nabla_{W} R\right)(X, Y) Z, \xi\right)=-g\left(\left(\nabla_{W} R\right)(X, Y) \xi, Z\right) .
$$

From (16) and (15), we have

$$
\left(\left(\nabla_{W} R\right)(X, Y) Z\right)=-g\left(\left(\nabla_{W} R\right)(X, Y) \xi, Z\right) \xi .
$$

Differentiating (8) and with the help of (6), we obtain

$$
\left(\nabla_{W} R\right)(X, Y) \xi=-g(\phi W, Y) X+g(X, \phi W) Y+R(X, Y) \phi W
$$


G. Ingalahalli, C.S. Bagewadi - A study on $\phi$-symmetric $\tau$-curvature tensor ...

By using (18) in (17), we get

$$
\begin{aligned}
\left(\left(\nabla_{W} R\right)(X, Y) Z\right) & =\{g(\phi W, Y) g(X, Z)-g(X, \phi W) g(Y, Z) \\
& -g(R(X, Y) \phi W, Z)\} \xi
\end{aligned}
$$

Again, if (19) holds, then (16) and (18) implies that the manifold is globally $\phi$ symmetric.

Thus, we can state the following:

Theorem 1. A K-contact manifold is globally $\phi$-symmetric if and only if the relation (19) holds for any vector fields $X, Y, Z$ and $W$ tangent to $M$.

Next, putting $Z=\xi$ in (17) and by virtue of (16), we have

$$
\left(\nabla_{W} R\right)(X, Y) \xi=0
$$

for any vector fields $X, Y, Z, W$ tangent to $M$. From (20) and (19), we get

$$
R(X, Y) \phi W=g(\phi W, Y) X-g(X, \phi W) Y .
$$

From (21), we get

$$
R(X, Y) W=g(W, Y) X-g(X, W) Y .
$$

Thus, the manifold is of constant curvature. Hence, we state the following theorem:

Theorem 2. A globally $\phi$-symmetric K-contact manifold is a space of constant curvature.

\section{Globally $\phi$ - $\tau$-Symmetric K-COntact manifold}

In this section, we define globally $\phi$ - $\tau$-symmetric K-contact manifold by

$$
\phi^{2}\left(\left(\nabla_{W} \tau\right)(X, Y) Z\right)=0,
$$

for all arbitrary vector fields $X, Y, Z, W$ on $M$.

From (2) and (23), we have

$$
-\left(\left(\nabla_{W} \tau\right)(X, Y) Z\right)+\eta\left(\left(\nabla_{W} \tau\right)(X, Y) Z\right) \xi=0 .
$$

By taking an inner product with respect to $U$, we get

$$
-g\left(\left(\nabla_{W} \tau\right)(X, Y) Z, U\right)+\eta\left(\left(\nabla_{W} \tau\right)(X, Y) Z\right) g(\xi, U)=0
$$


G. Ingalahalli, C.S. Bagewadi - A study on $\phi$-symmetric $\tau$-curvature tensor ...

Let $\left\{e_{i}: i=1,2, \ldots, 2 n+1\right\}$ be an orthonormal basis of the tangent space at any point of the manifold. Putting $X=U=e_{i}$ in (25) and taking summation over $i$, we get

$$
-g\left(\left(\nabla_{W} \tau\right)\left(e_{i}, Y\right) Z, e_{i}\right)+\eta\left(\left(\nabla_{W} \tau\right)\left(e_{i}, Y\right) Z\right) g\left(\xi, e_{i}\right)=0,
$$

with the help of (1) and on simplification, we obtain

$$
\begin{aligned}
& -\left[a_{0}+(2 n+1) a_{1}+a_{2}+a_{3}\right]\left(\nabla_{W} S\right)(Y, Z)-\left[a_{4}+2 n a_{7}\right]\left(\nabla_{W} r\right) g(Y, Z) \\
& -\quad a_{5} g\left(\left(\nabla_{W} Q\right) Y, Z\right)-a_{6} g\left(\left(\nabla_{W} Q\right) Z, Y\right)+a_{0} \eta\left(\left(\nabla_{W} R\right)(\xi, Y) Z\right)+a_{1}\left(\nabla_{W} S\right)(Y, Z) \\
& +a_{2}\left(\nabla_{W} S\right)(\xi, Z) \eta(Y)+a_{3}\left(\nabla_{W} S\right)(Y, \xi) \eta(Z)+a_{4} g(Y, Z) \eta\left(\left(\nabla_{W} Q\right) \xi\right) \\
& +a_{5} \eta(Z) \eta\left(\left(\nabla_{W} Q\right) Y\right)+a_{6} \eta(Y) \eta\left(\left(\nabla_{W} Q\right) Z\right)+a_{7}\left(\nabla_{W} r\right)[g(Y, Z)-\eta(Y) \eta(Z)](2 \emptyset)
\end{aligned}
$$

Putting $Z=\xi$ in (27) and on simplification, we get

$$
\left(\nabla_{W} S\right)(Y, \xi)=\frac{\left[a_{4}+2 n a_{7}\right]\left(\nabla_{W} r\right)}{\left[-a_{0}-2 n a_{1}-a_{2}-a_{6}\right]} \eta(Y),
$$

if $Y=\xi$ in (28), we get

$$
\frac{\left[a_{4}+2 n a_{7}\right]\left(\nabla_{W} r\right)}{\left[-a_{0}-2 n a_{1}-a_{2}-a_{6}\right]}=0 .
$$

The above equation (29) implies that $\frac{\left[a_{4}+2 n a_{7}\right]}{\left[-a_{0}-2 n a_{1}-a_{2}-a_{6}\right]} \neq 0$,

$$
\left(\nabla_{W} r\right)=0 \Longrightarrow r \text { is constant. }
$$

From (30) and (28), we have

$$
\left(\nabla_{W} S\right)(Y, \xi)=0
$$

we know that

$$
\left(\nabla_{W} S\right)(Y, \xi)=\nabla_{W} S(Y, \xi)-S\left(\nabla_{W} Y, \xi\right)-S\left(Y, \nabla_{W} \xi\right) .
$$

By using (6) and (10) in (32), we get

$$
\left(\nabla_{W} S\right)(Y, \xi)=S(Y, \phi W)-2 n g(Y, \phi W) .
$$

From (33) and (31), we have

$$
S(Y, \phi W)=2 n g(Y, \phi W) .
$$

Replacing $W=\phi W$ in (34), we have

$$
S(Y, W)=2 n g(Y, W) .
$$

Hence we can state the following:

Theorem 3. A globally $\phi$ - $\tau$-symmetric $K$-contact manifold is an Einstein manifold. 
G. Ingalahalli, C.S. Bagewadi - A study on $\phi$-symmetric $\tau$-curvature tensor ...

\section{5. $\tau$-RiCCI $\eta$-PARALLEL K-CONTACT MANIFOLD}

In this section, we examine the notion of $\tau$-Ricci $\eta$-parallelity for a K-contact manifold. At first, we give the definition of $\tau$-Ricci $\eta$-parallelity:

Definition 5. The $\tau$-Ricci tensor of a K-contact manifold is said to be $\eta$-parallel if it satisfies

$$
\left(\nabla_{X} S_{\tau}\right)(\phi Y, \phi Z)=0 .
$$

for all vector fields $X, Y, Z$.

From, (1) we have

$$
S_{\tau}(Y, Z)=\left[a_{0}+(2 n+1) a_{1}+a_{2}+a_{3}+a_{5}+a_{6}\right] S(Y, Z)+\left[a_{4}+2 n a_{7}\right] g(Y, Z) .
$$

Replacing $Y=\phi Y$ and $Z=\phi Z$, then we have

$$
\begin{aligned}
S_{\tau}(\phi Y, \phi Z) & =\left[a_{0}+(2 n+1) a_{1}+a_{2}+a_{3}+a_{5}+a_{6}\right] S(\phi Y, \phi Z) \\
& +\left[a_{4}+2 n a_{7}\right] \operatorname{rg}(\phi Y, \phi Z) .
\end{aligned}
$$

Differentiating (38) with respect to $X$, we get

$$
\begin{aligned}
\left(\nabla_{X} S_{\tau}\right)(\phi Y, \phi Z) & =\left[a_{0}+(2 n+1) a_{1}+a_{2}+a_{3}+a_{5}+a_{6}\right]\left(\nabla_{X} S\right)(\phi Y, \phi Z) \\
& +\left[a_{4}+2 n a_{7}\right]\left(\nabla_{X} r\right) g(\phi Y, \phi Z) .
\end{aligned}
$$

Again, differentiating (11) and by virtue of (5), we obtain

$$
\begin{aligned}
\left(\nabla_{X} S\right)(\phi Y, \phi Z) & =\left(\nabla_{X} S\right)(Y, Z)+2 n[g(\phi X, Y) \eta(Z)+g(\phi X, Z) \eta(Y)] \\
& +\eta(Y) S(X, \phi Z)+\eta(Z) S(\phi Y, X)
\end{aligned}
$$

By using (40) in (39), we have

$$
\begin{aligned}
\left(\nabla_{X} S_{\tau}\right)(\phi Y, \phi Z) & =\left[a_{0}+(2 n+1) a_{1}+a_{2}+a_{3}+a_{5}+a_{6}\right]\left\{\left(\nabla_{X} S\right)(Y, Z)+2 n[g(\phi X, Y) \eta(Z)\right. \\
& +g(\phi X, Z) \eta(Y)]+\eta(Y) S(X, \phi Z)+\eta(Z) S(\phi Y, X)\} \\
& +\left[a_{4}+2 n a_{7}\right]\left(\nabla_{X} r\right) g(\phi Y, \phi Z) .
\end{aligned}
$$

If $\left(\nabla_{X} S_{\tau}\right)(\phi Y, \phi Z)=0$, we get

$$
\begin{aligned}
\left(\nabla_{X} S\right)(Y, Z) & =-\frac{\left[a_{4}+2 n a_{7}\right]\left(\nabla_{X} r\right)}{\left[a_{0}+(2 n+1) a_{1}+a_{2}+a_{3}+a_{5}+a_{6}\right]} g(\phi Y, \phi Z) \\
& -2 n[g(\phi X, Y) \eta(Z)+g(\phi X, Z) \eta(Y)] \\
& -\eta(Y) S(X, \phi Z)-\eta(Z) S(\phi Y, X) .
\end{aligned}
$$

Hence we can state the following: 
G. Ingalahalli, C.S. Bagewadi - A study on $\phi$-symmetric $\tau$-curvature tensor ...

Theorem 4. A K-contact manifold is $\tau$-Ricci $\eta$-parallel if and only if the equation (42) holds with $\left[a_{0}+(2 n+1) a_{1}+a_{2}+a_{3}+a_{5}+a_{6}\right] \neq 0$.

Now, let $\left\{e_{i}: i=1,2, \ldots,(2 n+1)\right\}$, be an orthonormal basis of the tangent space at any point. Taking $Y=Z=e_{i}$ in (42) and then taking summation over $i$, we get

$$
\begin{aligned}
\left(\nabla_{X} S\right)\left(e_{i}, e_{i}\right) & =-\frac{\left[a_{4}+2 n a_{7}\right]\left(\nabla_{X} r\right)}{\left[a_{0}+(2 n+1) a_{1}+a_{2}+a_{3}+a_{5}+a_{6}\right]} g\left(\phi e_{i}, \phi e_{i}\right) \\
& -2 n\left[g\left(\phi X, e_{i}\right) \eta\left(e_{i}\right)+g\left(\phi X, e_{i}\right) \eta\left(e_{i}\right)\right] \\
& -\eta\left(e_{i}\right) S\left(X, \phi e_{i}\right)-\eta\left(e_{i}\right) S\left(\phi e_{i}, X\right) .
\end{aligned}
$$

On simplification of (43), we get

$$
\left[1+\frac{(2 n+1)\left[a_{4}+2 n a_{7}\right]}{\left[a_{0}+(2 n+1) a_{1}+a_{2}+a_{3}+a_{5}+a_{6}\right]}\right]\left(\nabla_{X} r\right)=0 .
$$

So we have $\left(\nabla_{X} r\right)=0$, which implies $r$ is constant, where $r$ is the scalar curvature of the manifold $M$. Hence we state the following theorem:

Theorem 5. If a K-contact manifold is $\tau$-Ricci $\eta$-parallel, then the scalar curvature is constant.

\section{REFERENCES}

[1] D.E. Blair, Contact manifolds in Riemannian geometry, Lecture Notes in Mathematics, Vol.509. Springer-Verlag, berlin-New-York, 1976.

[2] D.E. Blair, T. Koufogiorgos and B.J. Papantoniou, Contact metric manifolds satisfying a nullity condition, Israel J. Math., 91, (1995), 189-214.

[3] E. Boeckx, P. Buecken and L. Vanhecke, ф-symmetric contact metric spaces, Glasgow Math. J., 41, (1999), 409-416.

[4] U.C. De, A. A. Shaikh and S. Biswas, On ф-recurrent Sasakian manifolds, Novi Sad J. Math., 33, (2003), 13-48.

[5] U.C. De and Abdul Kalam Gazi, On $\phi$-recurrent $N(k)$-contact metric manifolds, Math. J. Okayama Univ., 50, (2008), 101-112.

[6] Gurupadavva Ingalahalli and C.S. Bagewadi, On $\phi$-symmetric $\tau$-curvature tensor in N(K)-Contact metric manifold, Carpathian Math. Publ. 6, 2 (2014), 203-211.

[7] M. Kon, Invariant submanifolds in Sasakian manifolds, Math. Ann., 219, (1975), 277-290. 
G. Ingalahalli, C.S. Bagewadi - A study on $\phi$-symmetric $\tau$-curvature tensor ...

[8] A.A. Shaikh and S.K. Hui, On Locally $\phi$-Symmetric $\beta$-Kenmotsu Manifolds, Extracta Mathematicae 24, 3 (2009), 301-316.

[9] M.M. Tripathi and Punam Gupta, $\tau$-curvature tensor on a semi-Riemannian manifold, J. Adv. Math. Stud., 4, 1 (2011), 117-129.

[10] M.M. Tripathi and Punam Gupta, On $\tau$-curvature tensor in K-contact and Sasakian manifolds, International Electronic Journal of Geometry, 4, (2011), 32-47.

[11] M.M. Tripathi and Punam Gupta, $(N(k), \xi)$-semi-Riemannian manifolds:Semisymmetries, arXiv:1202.6138v[math.DG]28(Feb 2012).

[12] H.G. Nagaraja and G. Somashekhara, $\tau$-curvature tensor in $(k, \mu)$-contact manifolds, Proceedings of the Estonian Academy of Sciences, 61, 1 (2012), 20-28.

[13] S. Tanno, Ricci curvatures of contact Riemannian manifolds, Tohoku Math. J., 40, (1988), 441-448.

[14] T. Takahashi, Sasakian ф-symmetric spaces, Tohoku Math. J., 29, (1977), 91113.

Gurupadavva Ingalahalli

Department of Mathematics,

Kuvempu University,

Shankaraghatta - 577 451, Shimoga, Karnataka, INDIA

email: gurupadavva@gmail.com

C.S. Bagewadi

Department of Mathematics,

Kuvempu University,

Shankaraghatta - 577 451, Shimoga, Karnataka, INDIA

email: prof_bagewadi@yahoo.co.in 